\title{
Renksiz Kaynağın Pasif Optik Ăg Sisteminde Veri Silme Analizi
}

\author{
Nisa NACAR ÇIKAN*1, Murat AKSOY1 \\ ${ }^{1}$ Çukurova Üniversitesi, Mühendislik Fakültesi, Elektrik Elektronik Mühendisliği Bölümü,, \\ Adana
}

Geliş tarihi: 14.12 .2020

Kabul tarihi: 31.03 .2021

Öz

Dalga boyu bölmeli çoğullamalı pasif optik ağlarda (DBBÇ POA) yeniden modülasyon tekniğinin uygulanması geniş bant kapasitesi, hızlı veri iletimi yanında sunucu ve kullanıcılar için oldukça uygun maliyet sağlamaktadır. Bu çalışmada yansıtıcı yarı iletken optik yükselteçlerin DBBÇ POA'larda 5 Gb/s hızında veri silme analizi birçok parametre incelenerek yapılmıştır. Kurulan sistemin optimum çalışma aralığını belirleyebilmek için enjekte gücü $-20 \mathrm{dBm}-0 \mathrm{dBm}$ arasında, ön gerilim akımı $20 \mathrm{~mA}-100 \mathrm{~mA}$ değerleri arasında incelenmiştir. Kalan sonlanma oranının 0,5 değeri ve altına düşmesini sağlayan sistem parametre değerleri belirlenerek renksiz kaynağın indirme yönünden kalan veriyi ne kadar silebildiği gösterilmiştir. Uygulanan renksiz kaynağın sistemi basitleştirerek birçok avantajı gösterilmiş ve daha önce uygulanmış olan Fabry Perot Laser Diyota alternatif olabileceği gösterilmiştir.

Anahtar Kelimeler: Reksiz kaynak, Veri silme, Pasif optik ağlar, Yeniden modülasyon

\section{Data Deletion Analysis of Colorless Source in Passive Optical Network System}

\begin{abstract}
The application of the remodulation technique in wavelength division multiplexed passive optical networks (WDM PON) provides a very affordable cost for the server and users as well as broadband capacity and fast data transmission. In this study, data deletion analysis of reflective semiconductor optical amplifiers in WDM PON at a speed of $5 \mathrm{~Gb} / \mathrm{s}$ is performed by examining many parameters. In order to determine the optimum operating range of the system, the injected power is applied between $-20 \mathrm{dBm}-0 \mathrm{dBm}$, and the bias current between $20 \mathrm{~mA}-100 \mathrm{~mA}$. By determining the system parameter values that allow the remained extinction ratio under 0.5 , it has been shown how much the colorless source can delete the remaining data at user end. It is demonstrated that how the applied colorless source simplifies the system and it can be an alternative to the previously applied Fabry Perot Laser Diode.
\end{abstract}

Keywords: Colorless source, Data erasing, Passive optical network, Remodulation

"Sorumlu yazar (Corresponding author): Nisa NACAR ÇIKAN, ncikan@cu.edu.tr 


\section{GIiRiş}

Son yıllarda geniş bant kullanımı ve yüksek veri oranı gerektiren uygulamalar için katlanarak artan bir talep vardır. Yüksek çözünürlük gerektiren televizyonlar, çevrimiçi oyunlar vb. gibi yüksek bant genişliği ve yüksek bilgi iletimi gerektiren sistemlerin artışı geleneksel geniş bant erişism teknolojilerinden (xDSL gibi), yeni nesil optik erişim ağlarına geçmeyi zorunlu kılmıştır. Yeni nesil haberleşme sistemlerinde fiber optik kablo kullanılarak, yüksek bant genişliğine ve yüksek veri hızlarına ulaşmak mümkün olmuştur.

Pasif optik ağlar (POA), erişim ağlarında yüksek bant genişliği ihtiyacını karşılamak amacıyla geliştirilmiştir. POA'lar arasında dalga boyu bölmeli çoğullamalı pasif optik ağ (DBBÇ POA) ise giderek artan bant genişliği ve veri hızı ihtiyacını karşılamak için nihai sonuç olarak önerilmektedir [1]. DBBÇ POA uygulamalarındaki en kritik problem kullanıcı tarafında düşük maliyette vericiler uygulamaktır [2,3]. $\mathrm{Bu}$ problemi çözmek ve operasyon harcamalarını azaltmak için renksiz kaynaklar kullanılmaktadır [4-8]. Renksiz kaynak uygulamasında birçok metot vardır ancak bunları temel olarak ikiye ayırabiliriz. Bunlar spektrum dilimleme ve optik geri dönüşüm çözümleridir [9-12]. Dalga boyu filtrelenmiş geniş optik spektrum 1şık kaynağı, spektrum dilimleme yöntemindeki her bir optik ağ birimi (OAB) için kullanılır. OAB'ler optik hat terminali (OHT)'lerde üretilen optik taşıyıcılarla veya ağın bir noktasında optik geri döngü yöntemi ile uzaktan beslenir. Genellikle, optik geri döngü yönteminde; OAB'lerin yansitıcı yüzleri vardır $[12,13]$. Fabry Perot Lazer Diyot (FPLD), ve yansıtıcılı yarı iletken optik yükselteç (YYOY)'ler, OAB'lerdeki yüksek veri hızlarını karşılamak için optik kaynaklar olarak kullanılabilir [4,11,12]. OAB'yi uzaktan besleyebilmek için indirme yönünde ayrı bir dalga boyu gönderilir. Böylece, OAB'ler yükleme yönünde belirli bir dalga boyu ile iletimi gerçekleştirir. Yükleme ve indirme yönleri için farklı dalga boylarının kullanılması, yüksek oranda alıc1-verici gerektirir ve ayrıca maliyetin artmasına neden olur. Başka bir metotta yükleme yönündeki bilgi, indirme yönünde bilgi taşıyan dalga boyu ile iletilebilir. $\mathrm{Bu}$ metot yeniden modülasyon tekniğidir [14-17]. Yeniden modülasyon metodunda indirme ve yükleme yönleri için aynı dalga boyunun kullanılması fiziksel bozulmalara neden olur [9]. Bu problemi giderebilmek için iki çözüm sunulmuştur. İlk çözüm olarak, ortogonal modülasyon biçimlerinin çift yönlü fiberde kullanılmasıdır [18-20]. Fakat merkez ofis ve kullanıcı tarafında farklı modülasyon tekniklerinin kullanımı maliyeti oldukça arttırmaktadır [14]. İkinci çözüm olarak ise, indirme yönündeki verinin silinerek, yükleme yönündeki verinin merkez ofisten gelen aynı taşıyıcı dalga ile taşınmasıdır [21]. Bu nedenle; veri silme mekanizması gelecekteki optik erişim ağları için umut vaat eden uygun maliyetli bir seçenektir [22,23].

Bu çalışmada geniş bant kapasitesine sahip ve yüksek hızlarda iletim yapılmasını sağlayan DBBÇ POA'lar ile kurulan sistemin uygun maliyette sunulmasını sağlayabilmek için kullanıcı tarafında renksiz kaynak olarak YYOY kullanılmıştır. Aynı zamanda DBBÇ POA'larda uygulanan yeniden modülasyon tekniği düşük maliyeti ile yüksek hızlarda, geniş bant genişliğinde, uzun mesafelerde düşük kayıpla, son kullanıcı ve operatörler açısından çok önemli olan fiyat problemini düşürerek veri iletimini sağlamaktadır. DBBÇ POA'larda son kullanıcı tarafında FPLD uygulanarak veri silme analizi daha önceki çalışmalarda incelenmiştir [14-16]. [16]'de sistemde veri silme analizi incelenirken sadece ön gerilim akımı ve enjekte edilen güç olmak üzere sadece iki parametre göz ününe alınmıştır. Fakat sistemi etkileyen diğer birçok parametre incelenmemiştir. [14]'te sisteme etkisi olan ve fiber iletimde mutlaka değerlendirilmesi gereken hapsetme faktörü, sonlanma oranı akım seviyeleri, enjekte edilen güç ve yüksek yansıtma yüzeyine sahip olan FPLD üzerinde çalışılmıştır. [15]'de ise literatürde daha önce tartışılmamış olan zayıf rezonant kaviteli bir FPLD'nin yeniden modülasyon sisteminde renksiz kaynak olarak uygulandığında ön yüz yansitıcılığının etkileri üzerinde çalışılmış ve $\% 7$ yüz yansıtma değerinin 
en iyi sonucu verdiği gösterilmiştir. [24]'de modülasyon ortalama tekniği kullanılmış fakat bu teknikte ortalama genliği bulabilmek için birçok fiber döngüsü kullanılmış ve buda sistem maliyetini artırmaktadır. Ayrıca sistem hızı olarak $1,25 \mathrm{~Gb} / \mathrm{s}$ belirlenmiştir ve sistemi etkileyen birçok parametre göz önüne alınmamıştır. Önerilen hız seviyesi hızla artan bant genişliğini talebini ileride karşılamayabilmektedir. [25]'de sistemin bir tarafında kendinden beslemeli teknik uygulandığından, aynı zamanda sistemin her iki ucunda da YYOY uygulanmasindan dolayı sistem bant genişliği sınırlandırılmıştır. [26]'da sistemin iki tarafında maliyeti oldukça arttıran farklı modülasyon teknikleri uygulanmış, ayrıca çift yönlü iletimde avantaj sağlayan veri silme analizi uygulanmamıştır. Bu çalışmada, çift yönlü bir fiber iletişimde, yeniden modülayon tekniği uygulanarak renksiz kaynak olarak FPLD kadar etkili ve bir alternatif oluşturacak YYOY'nin veri silme analizi birçok faktör göz önüne alınarak ve Optisystem 15 yazılım programı kullanarak çalışılmıştır. Sistem hızı, giderek artan bant genişliği ihtiyacını karşılayabilecek olan $5 \mathrm{~Gb} / \mathrm{s}$ olarak belirlenmiştir. Hapsetme oranı kabul edilir oranda artırıldığında sistemde oluşturduğu pozitif etkiler gösterilmiştir. Elde edilen sonuçlar önerilen sistemde YYOY'nin uygulanması ile yüksek veri hızlarında, uygun maliyetli, iyi sonuçlar elde edilebileceğini göstermiştir ve gelecek çalışmalar açısından umut vericidir.

\section{MATERYAL VE METOT}

Şekil 1 kullanıcı tarafında renksiz kaynak olarak YYOY kullanan bir yeniden modülasyon modelini göstermektedir. Sistem OHT, iletim birimi ve kullanıcı tarafı olmak üzere üç bölümden oluşmaktadır. Sistemde ilk olarak, indirme yönündeki sıfıra dönüşü olmayan (NRZ) veri Mach-Zehnder modülatör (MZM) kullanılarak modüle edilmiştir. Sistemde uygulanan dizi dalga kılavuzu ızgarası (DDKI), verilerin çoğullama ve çözümüne yardımcı olur. OAB tarafındaki optik bağlayıcı indirme yönünden gelen sinyali ikiye böler. Düşük yoğunluktaki güç sinyali alıcıya gönderilirken, yüksek yoğunluktaki sinyal OAB'deki vericiye gönderir. Böylece sistemde indirme ve yükleme olmak üzere her iki yönde iletim için aynı taşıyıcı kullanılmaktadır.

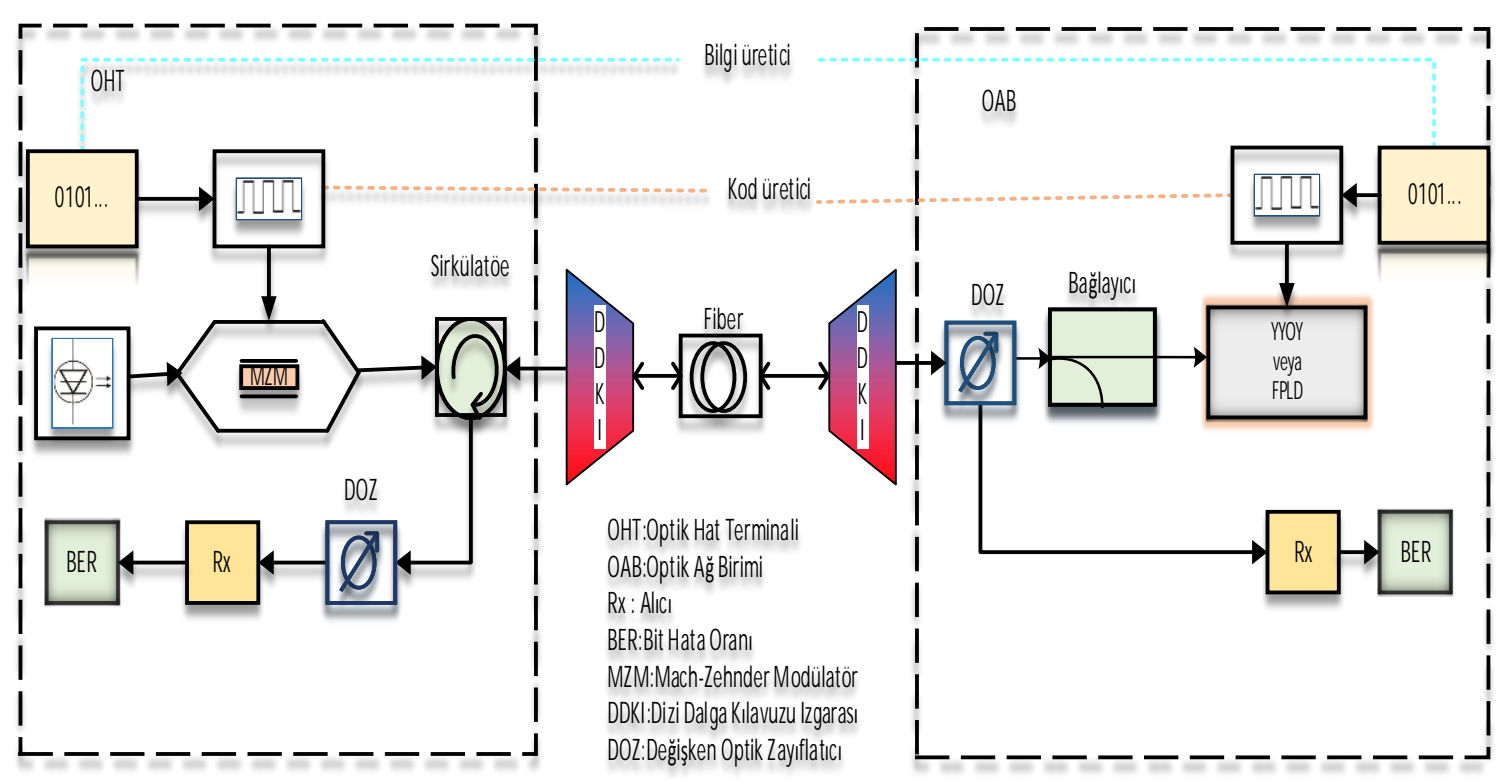

Şekil 1. Yeniden modülasyon POA sistemi 
Sistemde indirme yönündeki taşıyıcı üzerindeki kalan verinin silinerek, aynı taşıyıcının yükleme yönündeki veriyi iletebilmesi için tekrar kullanması amaçlanmıştır. $\mathrm{Bu}$ sebeple, indirme yönünden gelerek hala taşıyıcı üzerinde kalan sinyal renksiz optik kaynak tarafından silinmelidir. Yükleme ve indirme yönündeki verileri ayırmak için sirkülatör kullanılmıştır. Sistemde kullanılan değişken optik zayıflatıcı (DOZ) yardımıyla ise güç seviyesi ayarlanmaktadır. OAB ve OHT bölümlerinde yer alan bit hata oranı (BER) cihazları ile hem veri iletim kalitesi hem de YYOY tarafından silinen verinin analizi yapılmıştır.

Sistemde indirme yönünde sonlanma oranı $\left(\mathrm{SO}_{\mathrm{i}}\right)$ $5 \mathrm{~dB}$ ve $10 \mathrm{~dB}$ olarak uygulanmıştır. Modüle edilmiş sinyal OAB'ye gönderilir ve tekrar yansitilarak OHT' ye gelir. Yansitılan sinyalin veri silme analizi, belirli parametrelerdeki değişiklikler göz önüne alınarak incelenmiştir. Güç değeri -20 dB' den başlayarak, 0 dB'ye ulaşıncaya kadar kabul edilen belirli aralıklarda incelenmiştir. Tasarımın veri hızı 193,1 THz penceresinde 5 Gb/s'dir. YYOY'ya ait önemli spesifik parametreler Tablo 1'de verilmiştir.

Tablo 1. YYOY parametreleri

\begin{tabular}{|l|c|c|}
\hline Parametre & Değeri & Birimi \\
\hline Giriş eşleşme kaybı & 3 & $\mathrm{~dB}$ \\
\hline Çıkış eşleşme kaybı & 3 & $\mathrm{~dB}$ \\
\hline Giriş yüzey yansıtıcılığı & $50 \mathrm{e}-06$ & \\
\hline Çıkış yüzey yansıtıcılığ1 & 0.99 & \\
\hline Aktif uzunluk & $600 \mathrm{e}-06$ & $\mathrm{~m}$ \\
\hline Genişlik & $0.4 \mathrm{e}-06$ & $\mathrm{~m}$ \\
\hline Optik hapsolma faktörü & 0.4 & \\
\hline
\end{tabular}

\section{SONUÇLAR VE TARTIŞMA}

Ön gerilim akımına karşı kazanç grafiği ve enjeksiyon gücüne karşı kazanç grafiği sırasıyla Şekil 2a ve Şekil 2b'de gösterilmektedir. $25 \mathrm{~dB}$ enjekte edilen güç ve $100 \mathrm{~mA}$ ön gerilim akımı için elde edilen kazanç değeri, -22 dB'dir. Kazanç, 60 mA'nın üzerinde doymaya başlamıştır. Ayrıca,
Şekil 2b'de görüleceği gibi kazanç $-20 \mathrm{dBm}$ enjekte güç üzerinde doyum noktasına ulaşmıştır.

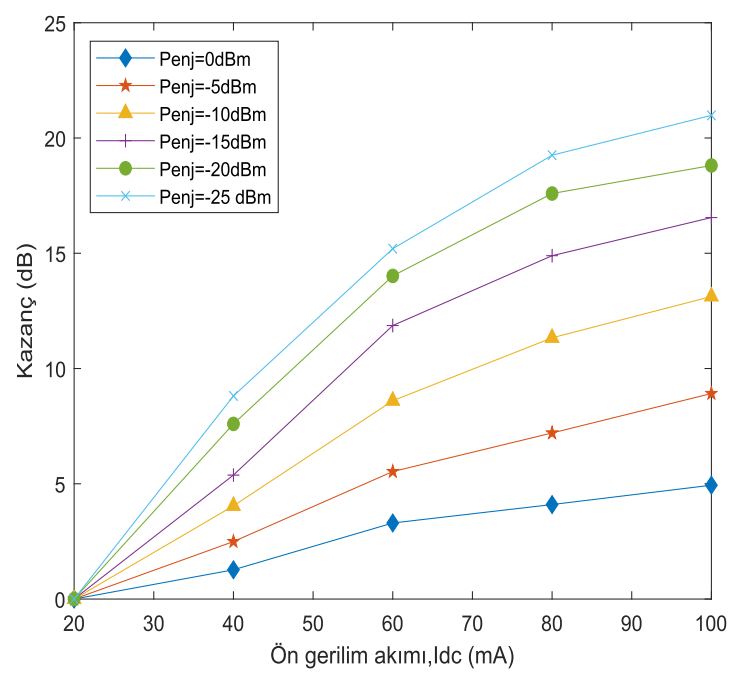

(a)

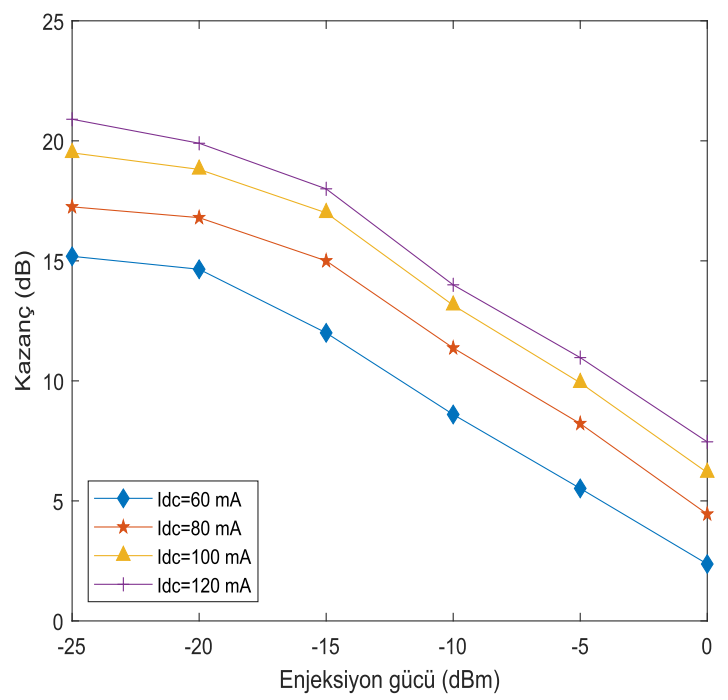

(b)

Şekil 2. YYOY kazanç grafiği a. Ön gerilim akımına göre, b. Enjeksiyon gücüne göre

Ön gerilim akımına göre indirme yönünde sonlanma oranı grafiği Şekil 3'te gösterildiği gibidir. $\mathrm{Bu}$ grafikte, aralarında karşılaştırma yapmak için iki farklı sonlanma oranı ve enjekte edilen güç değerleri simüle edilmiştir. 


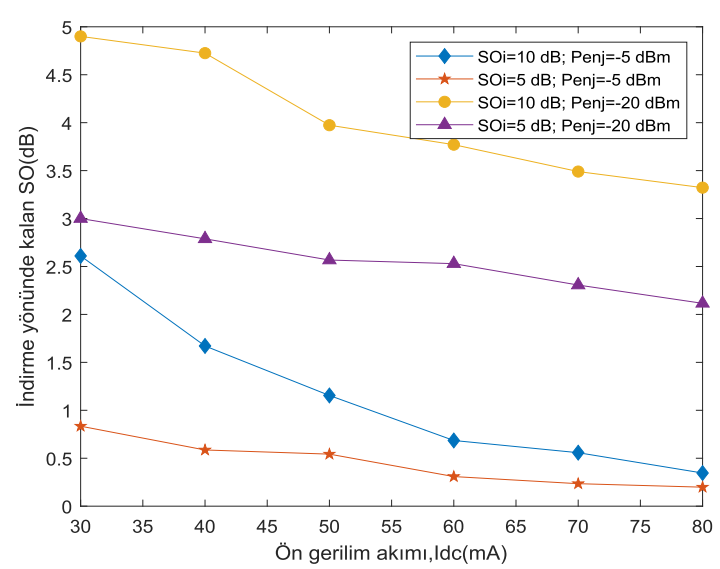

Şekil 3. Farklı değerlerde sonlanma oranına karşı ön gerilim akım grafiği

$\mathrm{SO}_{\mathrm{i}}$ 'nin azaltılmasının ve Penj değerlerinin artırılmasının artık verilerin tamamen silinmesine yardımcı olduğu gözlemlenmektedir. 0,5 değerinin altındaki $\mathrm{SO}_{\mathrm{i}}$ değeri verilerin silindiğini gösterir. $\mathrm{SO}_{\mathrm{i}}$ değerinin $5 \mathrm{~dB}$ ve Penj değerinin $-5 \mathrm{dBm}$ olduğu durumda kalan artık verinin tamamen silindiği görülmektedir. Penj değeri $-20 \mathrm{dBm}$ 'de, çalışma bölgesinin doğrusal alanında olduğundan verileri silemez.

Ön gerilim akımının artırılması, artık verilerin silinebileceğini gösteren başka bir önemli faktördür. Ancak akımın artırılması, cihaz içinde oluşan mod rekabetini de artırır. Bu nedenle, artan akıma göre yükleme yönündeki BER grafiği Şekil 4'te gösterilmektedir.

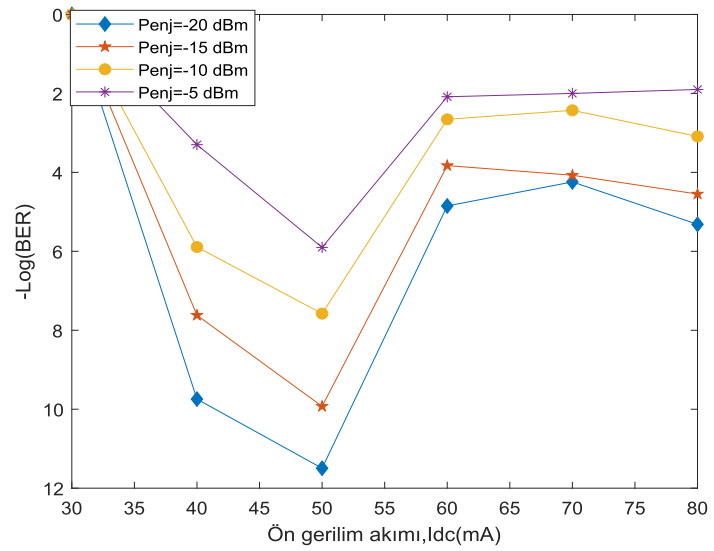

Şekil 4. BER\& ön gerilim akım grafiği
Şekil 4, YYOY'ya uygulanan ön gerilim akımının indirme yönünden kalan verileri silmek için 50 mA'dan büyük olması gerektiği şeklinde açıklanabilir.

Şekil 5, artan verinin -5 dBm enjeksiyon gücünde tamamen silinebildiğini göstermektedir. Güç -15 dBm'nin altında uygulandığında, merkez ofiste artan veri algılanır ve bu da verilerin OHT'de tam olarak silinmediğini gösterir.

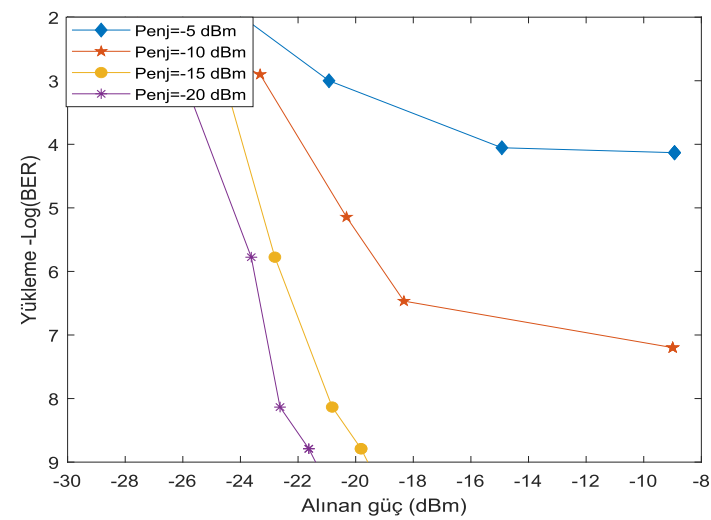

Şekil 5. BER \& alınan güç grafiği

Verilerin silinmesini etkileyen önemli bir diğer faktör ise hapsolma faktörüdür (HF). Hapsolma faktörünün artırılması, verilerin Şekil 6'da gösterildiği gibi kolayca silinmesine yardımcı olur. Enjeksiyon gücünün sabit bir hapsolma faktöründe arttırılması da kalan verilerin silinmesini etkileyen diğer bir nedendir.

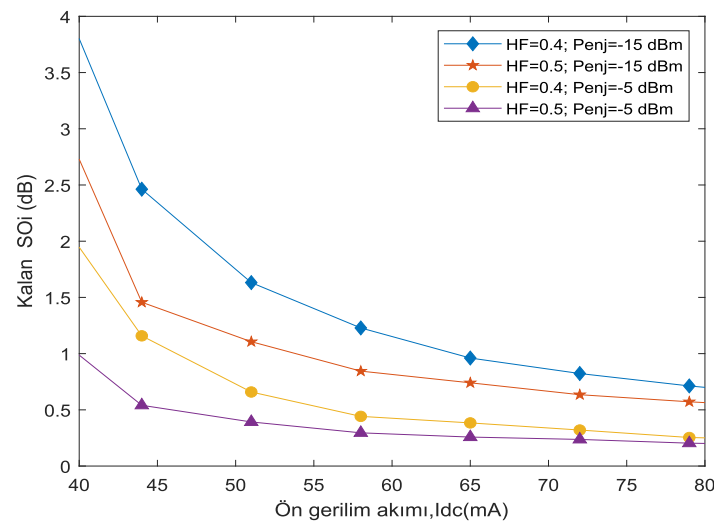

Şekil 6. Farklı HF ve Penj değerlerinde $\mathrm{SO}_{\mathrm{i}}$ \& ön gerilim akım grafiği 
HF değerinin 0,4'ten 0,5'e yükseltilmesi kalan verinin silinme oranını artırır. Aynı şekilde Penj değerini $-15 \mathrm{dBm}$ 'den $-5 \mathrm{dBm}$ 'e yükseltilmesi de kalan verinin tam olarak silinmesinde önemli bir rol oynar.

\section{SONUÇ}

$\mathrm{Bu}$ çalışmada, yeniden modülasyon tekniğinin uygulandığ 1 bir DBBÇ POA sistemine OAB tarafında renksiz kaynak olarak kullanılan YYOY'nin veri silme verimliliği analiz edilmiştir. Sistem incelenirken sonlanma oran1, hapsolma faktörü, farklı enjeksiyon güç seviyeleri, farklı ön gerilim akım seviyeleri, bit hata oranı gibi sistem çalışmasını etkileyen önemli parametreler dikkate alınmıştır. Sistem hızı 5 Gb/s'dır. YYOY'nın kalan veriyi silme analizinin yapılabilmesi için ön gerilim akımı $20 \mathrm{~mA}-100 \mathrm{~mA}$ aralığında, enjeksiyon gücü ise $-25 \mathrm{dBm}-0 \mathrm{dBm}$ aralığında incelenmiştir. Ön gerilim akımının artışıyla birlikte renksiz kaynağın veri silme verimliliğinin arttığ gösterilmiştir.

Parametre değerlerinden ön gerilim akımının $50 \mathrm{~mA}$, hapsolma faktörünün 0,4 ve enjeksiyon gücünün $-15 \mathrm{dBm}$ ve üzerinde olması OHT'lerde indirme ve yükleme yönündeki verilerin karışmasını önlemekte ve aynı zamanda renksiz kalan verinin renksiz kaynak tarafindan tam olarak silinmesini sağlamaktadır. Silme işlemi sayesinde çift fiber yerine, tek fiber kullanılarak sistemin maliyetinin düşürülmesi sağlanmıştır. Sisteme YYOY uygulandığında, uygulanması gereken optimum değerler daha önce çalışılmamış birçok parametre incelenerek belirlenmiştir ve böylece elde edilen sonuçlar, gelecek çalışmalar için yol göstericidir.

\section{KAYNAKLAR}

1. Antoniades, N.N., Ellinas, G., Roudas, I., 2011. WDM Systems and Networks: Modeling, Simulation, Design and Engineering: Springer New York, 532, doi: 10.1007/978-1-46141093-5.

2. Berrettini, G., Meloni, G., Giorgi, L., Ponzini, F., Cavaliere, F., Ghiggino, P., Bogoni, A.
2009. Colorless WDM-PON Performance Improvement Exploiting a Service-ONU for Multiwavelength Distribution. Paper Presented at the 2009 Conference on Optical Fiber Communication-incudes Post Deadline Papers.

3. Celino, D.R., Duarte, U.R., Romero, M.A., 2020. Improved Self-seeding and Carrier Remodulation Performance for WDM-PON by Means of Double RSOA Erasure. Optics Communications, 459, 125018. doi: https:// doi.org/10.1016/j.optcom.2019.125018.

4. Chi, Y., Lin, C., Lin, S., Lin, G., 2012. The Reuse of Downstream Carrier Data Erased by Self-Feedback SOA for Bidirectional DWDMPON Transmission. Journal of Lightwave Technology, 30(19), 3096-3102. doi:10.1109/ JLT.2012.2210698.

5. Cikan, N.N., Aksoy, M., 2019. A Review of Self-Seeded RSOA Based on WDM PON. Canadian Journal of Electrical and Computer Engineering, 42(1), 2-9. doi:10.1109/CJECE. 2018.2872433.

6. Cikan, N.N., Aksoy, M., 2020a. Data Erasure Analysis of FPLD Based on Remodulation Bidirectional PON System. Journal of Modern Optics, 67(2), 139-145. doi:10.1080/09500340. 2019.1699182.

7. Cikan, N.N., Aksoy, M., 2020b. Data Erasure in Fabry-Perot Diode Lasers: Effects of Facet Reflectivity. Journal of Modern Optics, 67(6), 515-522. doi:10.1080/09500340.2020.1759716

8. Hu, Z., Qiu, Y., Li, W., Chan, C.K., 2020. Experimental Demonstration of Direct Remodulation for an IM/DD OFDM-WDM-PON with Symmetrical Bi-directional Transmission. Optics Communications, 460, 125123. doi:https://doi.org/10.1016/j.optcom.2019.1251 23.

9. Kazovsky, L.G., Shaw, W., Gutierrez, D., Cheng, N., Wong, S., 2007. Next-generation Optical Access Networks. Journal of Lightwave Technology, 25(11), 3428-3442. doi:10.1109/JLT.2007.907748.

10. Ki-Man, C., Jin-Serk, B., Chang-Hee, L., 2006. Color-free Operation of Dense WDM-PON Based on the Wavelength-locked Fabry-Pe/spl Acute/rot Laser Diodes Injecting a Low-noise BLS. IEEE Photonics Technology Letters, 
18(10), 1167-1169. doi:10.1109/LPT.2006. 874719 .

11. Li, Y., Chi, Y., Tsai, C., Cheng, M., Lin, G. 2015. Reusing Downstream Carrier in Colorless Laser Diode for Full-Duplex 64QAM OFDM. Journal of Lightwave Technology, 33(9), 1780-1787. doi:10.1109/ JLT.2014.2384524.

12. Lin, G., Liao, Y., Chi, Y., Kuo, H., Lin, G., Wang, H., Chen, Y., 2010. Long-Cavity Fabry-Perot Laser Amplifier Transmitter with Enhanced Injection-Locking Bandwidth for WDM-PON Application. Journal of Lightwave Technology, 28(20), 2925-2932. doi:10.1109/ JLT.2010.2060470.

13. Lin, G., Wang, H., Lin, G., Huang, Y., Lin, Y., Cheng, T., 2009. Comparison on InjectionLocked Fabry-Perot Laser Diode with FrontFacet Reflectivity of $1 \%$ and $30 \%$ for Optical Data Transmission in WDM-PON System. Journal of Lightwave Technology, 27(14), 2779-2785. doi:10.1109/JLT.2009.2016676.

14. Liu, C., Ding, Q.A., Song, J., Zhang, L., Wang, X., Nie, B., Cheng, X., 2021. Performance Investigation of PM-based Wavelength Remodulation Scheme in Bidirectional TWDM-PON. Journal of Optical Communications. doi:doi:10.1515/joc-20200198.

15. Lopera, J.A., Cárdenas, A.M., Quintero, G.A., Zapata, J.D., Granada Torres, J.J., 2020. Lowreflective Fabry-Perot Laser Diode Cavity as a Colorless Source on a WDM-PON System. Paper Presented at the OSA Advanced Photonics Congress (AP) 2020 (IPR, NP, NOMA, Networks, PVLED, PSC, SPPCom, SOF), Washington, DC, NeTu2B.3.

16. Martinez, J.J., Gregorio, J.I. G., Lucia, A.L., Velasco, A.V., Aguado, J.C., Binue, M.Á.L., 2008. Novel WDM-PON Architecture Based on a Spectrally Efficient IM-FSK Scheme Using DMLs and RSOAs. Journal of Lightwave Technology, 26(3), 350-356. doi:10.1109/JLT.2007.909864.

17. Park, S., Choi, Y., Oh, J., Koo, S., Lee, D., 2007. An Evolution Scenario of a Broadband Access Network Using R-SOA-Based WDMPON Technologies. Journal of Lightwave
Technology, 25(11), 3479-3487. doi:10.1109/ JLT.2007.907789.

18. Payoux, F., Chanclou, P., Moignard, M., Brenot, R., 2005. Gigabit Optical Access Using WDM PON Based on Spectrum Slicing and Reflective SOA. Paper Presented at the 2005 $31^{\text {st }}$ European Conference on Optical Communication, ECOC 2005.

19. Prat, J., Polo, V., Bock, C., Arellano, C., Olmos, J.J.V., 2005. Full-duplex Single Fiber Transmission Using FSK Downstream and IM Remote Upstream Modulations for Fiber-tothe-home. IEEE Photonics Technology Letters, 17(3), 702-704. doi:10.1109/LPT.2004.840930.

20. Schrenk, B., Chatzi, S., Bonada, F., Lazaro, J. A., Klonidis, D., Tomkos, I., Prat, J., 2009. $\mathrm{C}+\mathrm{L}$ Band Remote Node for Amplification in Extended Reach Full-duplex 10Gb/s WDM/TDM Passive Optical Networks. Paper Presented at the $200935^{\text {th }}$ European Conference on Optical Communication, 1-2.

21. Šprem, M., Babić, D., 2019. Wavelength Reuse WDM-PON Using RSOA and Modulation Averaging. Optics Communications, 451, 1-5. doi:https://doi.org/10.1016/j.optcom.2019.06.0 24.

22. Su, Y., Chi, Y., Chen, H., Lin, G., 2015. Data Erasing and Rewriting Capabilities of a Colorless FPLD Based Carrier-reusing Transmitter. IEEE Photonics Journal, 7(3), 1-12. doi:10.1109/JPHOT.2015.2412457.

23. Takesue, H., Sugie, T., 2003. Wavelength Channel Data Rewrite Using Saturated SOA Modulator for WDM Networks with Centralized Light Sources. Journal of Lightwave Technology, 21(11), 2546-2556. doi:10.1109/JLT.2003.819532.

24. Wai, H., Chun-Kit, C., Lian-Kuan, C., Tong, F., 2003. An Optical Network Unit for WDM Access Networks with Downstream DPSK and Upstream Remodulated OOK Data Using Injection-locked FP Laser. IEEE Photonics Technology Letters, 15(10), 1476-1478. doi:10.1109/LPT.2003.818055

25. Yeh, C., Chien, H., Chi, S., 2008. CostEffective Colorless RSOA-Based WDM-PON with $2.5 \mathrm{Gbit} / \mathrm{s}$ Uplink Signal. Paper Presented at the OFC/NFOEC 2008-2008 Conference on 
Renksiz Kaynă̆ın Pasif Optik Ă̆ Sisteminde Veri Silme Analizi

Optical Fiber Communication/National Fiber Optic Engineers Conference.

26.Zuo, C., Li, X., 2020. PolarizationDiscriminated RSOA-EAM for Colorless Transmitter in WDM-PON. Applied Sciences, 10(24), 9049. Retrieved from https://www.mdpi.com/2076-3417/10/24/9049. 